TITLE:

A discrete-time approach to
stability analysis of systems with
aperiodic sample-and-hold devices

AUTHOR(S):

Fujioka, Hisaya

CITATION:

Fujioka, Hisaya. A discrete-time approach to stability analysis of systems with aperiodic sample-and-hold devices. IEEE Transactions on Automatic Control 2009, 54(10): 24402445

ISSUE DATE:

2009-10

URL:

http://hdl.handle.net/2433/87747

RIGHT:

c 2009 IEEE. 


\title{
A Discrete-Time Approach to Stability Analysis of Systems With Aperiodic Sample-and-Hold Devices
}

\author{
Hisaya Fujioka
}

\begin{abstract}
Motivated by the widespread use of networked and embedded control systems, an algorithm for stability analysis is proposed for sampleddata feedback control systems with uncertainly time-varying sampling intervals. The algorithm is based on the robustness of related discrete-time systems against perturbation caused by the variation of sampling intervals. The validity of the algorithm is demonstrated by numerical examples.
\end{abstract}

Index Terms-Matrix exponential, networked control systems, quadratic stability, sampled-data systems.

\section{INTRODUCTION}

The sampled-data control theory (See [1] and references therein) has been well-developed in the last two decades, where periodic sampling is assumed and resulting periodicity of the closed-loop systems plays a crucial role. It is indeed reasonable to consider the periodic sampling in

Manuscript received June 19, 2008; revised February 28, 2009 and May 22, 2009. First published September 22, 2009; current version published October 07, 2009. Recommended by Associate Editor P. Shi.

The author is with the Graduate School of Informatics, Kyoto University, Kyoto 606-8501, Japan (e-mail: fujioka@i.kyoto-u.ac.jp).

Color versions of one or more of the figures in this technical note are available online at http://ieeexplore.iee.org.

Digital Object Identifier 10.1109/TAC.2009.2029304 
the conventional implementation of sampled-data systems. In some recent applications, however, it is hard to perform the periodic sampling. For example, resources for measurement and control are restricted in networked and/or embedded control systems (See [2], [3] and references therein), and hence the sampling operation results to be aperiodic and uncertainly time-varying. In view of the widespread use of networked and/or embedded control systems, it is theoretically and practically important to study the robustness of such systems against variation of sampling intervals. Since the pioneering work [4]-[6], one can find several studies in the literature [7]-[14] for this issue. It is worth mentioning that most of existing results verify stability by showing the existence of a continuous-time Lyapunov function either explicitly [4], [5], [7]-[12] or implicitly [13], [14].

The purpose of this technical note is also to develop an algorithm to check the stability of the aperiodic sampled-data systems. We, however, will take a different approach: Once we fix a sampling interval in the given range, we have a time-invariant discrete-time system corresponding to the fixed interval. If there exists a quadratic discrete-time Lyapunov function which verifies stability of all such discrete-time systems corresponding to sampling intervals in the range, the exponential stability of the aperiodic sampled-data systems follows [6]. We will use this property to prove the stability. This approach is already considered in [6], [15]. They, however, approximate the set of all possible sampling intervals by a grid, i.e., a set of a finite number of sampling intervals. Although the approximation enables them to provide finite step procedures related to stability, they cannot conclude stability of the original systems.

This technical note will provide a procedure which can conclude stability of the original systems. For the purpose we will first study stability robustness of sampled-data systems against perturbation caused by the variation of sampling intervals, based on a small-gain modeling of the perturbation. Then the following property will be derived from the robustness: Supposing that there exists a discrete-time Lyapunov function which concludes the stability of a given original system as described above, there exists a grid such that the existence of a Lyapunov function shared on the grid does conclude the stability of the original system. We will also construct an algorithm which seeks such a grid.

This technical note is organized as follows: The problem is formulated in Section II. Section III provides a stability criteria and an algorithm to verify the stability based on the criteria. The validity of the algorithm is demonstrated in Section IV. We discuss directions to reduce the conservatism in Section V.

\section{PROBLEM Formulation}

Let the following state-space system be given

$$
\dot{x}(t)=A x(t)+B u(t)
$$

where $x$ and $u$ respectively denote the state and the input taking values in $\mathbb{R}^{n}$ and $\mathbb{R}^{m} . A$ and $B$ are matrices of compatible dimensions.

We consider the following scenario of the feedback control of (1):

- We can measure the state of (1) when $t=\tau_{k}(k=0,1, \ldots)$ where $\left\{\tau_{k}\right\}$ is an uncertain set of discrete time instances satisfying

$$
\tau_{0}=0
$$

and

$$
0<h_{\ell} \leq \tau_{k+1}-\tau_{k} \leq h_{u}<\infty
$$

for given $h_{\ell}$ and $h_{u}$.

- The control input $u$ is determined from the sampled-data $x\left(\tau_{k}\right)$ and a given feedback gain $F \in \mathbb{R}^{m \times n}$ by

$$
u(t)=F x\left(\tau_{k}\right), \quad \forall t \in\left[\tau_{k}, \tau_{k+1}\right) .
$$

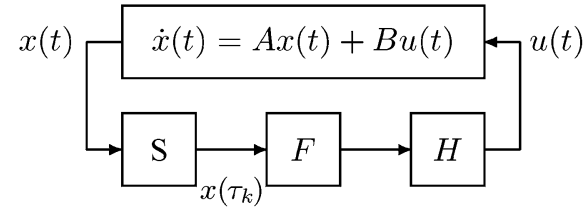

Fig. 1. Feedback control with aperiodic sample and hold actions.

The resulting feedback system composed of (1) and (4), denoted by $T$, is given by

$$
\dot{x}(t)=A x(t)+B F x\left(\tau_{k}\right), \quad \forall t \in\left[\tau_{k}, \tau_{k+1}\right) .
$$

See also Fig. 1 where $S$ and $H$ denote the sampler and the hold device respectively. Note that (3) implies

$$
\lim _{k \rightarrow \infty} \tau_{k}=\infty
$$

since $h_{\ell}>0$. Applications of this scenario can be found in networked and/or embedded control systems [2], [3], where resources for measurement and control are restricted.

Remark 1: There is a number of studies of networked control systems considering the transmission delays, e.g., [16]-[20]. They are related to this technical note since one can transform (4) to

$$
u(t)=F x(t-L(t)), \quad L(t)=t-\tau_{k}, \quad \forall t \in\left[\tau_{k}, \tau_{k+1}\right)
$$

as pointed out in [7]. Indeed stability of $T$ follows if the corresponding MATI (maximum allowable transfer interval) is greater than $h_{u}$. However, the delay corresponding to the non-uniform sampling is structured as shown above, and hence a direct application of results on systems with delay introduces conservatism as pointed out in [13].

Remark 2: We have assumed that $h_{\ell}>0$ in (3), which is not required in the methods based on continuous-time Lyapunov functions [4], [5], [7]-[14], [16], [17]. This is a fundamental limitation of the method to prove stability based on a discrete-time Lyapunov function which we will see below, although the case $h_{\ell}=0$, i.e., continuous measurement, would never happen in the implementation of networked/embedded control systems.

The purpose of this technical note is to provide stability criteria for $T$. If $\tau_{k}$ 's satisfy

$$
\tau_{k+1}-\tau_{k}=\tilde{h}
$$

for some $\tilde{h} \in\left[h_{\ell}, h_{u}\right]$, the resulting feedback control system is periodic. This special scenario is the one well-studied in the so-called sampled-data control theory [1]. Indeed the stability can be easily verified by checking the spectral radius of $\Phi(\tilde{h})$ in the special scenario, where

$$
\Phi(h):=\mathrm{e}^{A h}+\int_{0}^{h} \mathrm{e}^{A(h-\eta)} B \mathrm{~d} \eta F .
$$

It is, however, obvious that our general scenario is much more complicated, because of the uncertainly time-varying nature.

In this technical note we will verify the stability of $T$ based on the following lemma [3], [6], which can be proved by using the boundedness of $h_{u}$ :

Lemma 1: $T$ is exponentially stable if there exists a matrix $0<P=$ $P^{*} \in \mathbb{R}^{n \times n}$ satisfying

$$
(\Phi(h))^{*} P \Phi(h)-P<0
$$

for all $h \in\left[h_{\ell}, h_{u}\right]$, where $\Phi(\cdot)$ is defined in (6). 
Note that Lemma 1 is based on the quadratic stability of the accompanying discrete-time system $T_{d}$ defined by

$$
\xi[k+1]=\Phi\left(\tau_{k+1}-\tau_{k}\right) \xi[k]
$$

with the discrete-time Lyapunov function

$$
V(\xi[k]):=\xi^{*}[k] P \xi[k]
$$

where $\xi[k]:=x\left(\tau_{k}\right)$.

Note also that it is hard to find a matrix $P$ in Lemma 1 since the inequality (7) must hold for all values in $\left[h_{\ell}, h_{u}\right]$, and $\Phi$ is a strongly nonlinear function of $h$.

Zhang-Branicky [6] proposes a randomized algorithm to search a $P$ on a grid between $h_{\ell}$ and $h_{u}$. In other words, the algorithm in [6] determines if a necessary condition for a sufficient condition for the stability holds or not, and hence cannot conclude the stability. Similar idea of gridding is used in [15].

\section{MAIN RESULTS}

In the gridding methods [6], [15] we expect that the existence of $P>0$ satisfying (7) for all $h \in\left[h_{\ell}, h_{u}\right]$ is implied by that for all $h \in \mathcal{G}$, where $\mathcal{G}$ is a grid

$$
\mathcal{G}=\left\{h_{1}, h_{2}, \ldots, h_{N}\right\} \subset\left[h_{\ell}, h_{u}\right]
$$

The conjecture is not true in general. The basic idea of the stability analysis in this technical note is, however, to find a grid $\mathcal{G}$ for which the conjecture is true. In the sequel we first discuss the robustness of systems with uniform sampling interval against the perturbation caused by the variation of sampling interval. Then existence of such a grid is proved in a rigorous manner, and an algorithm to generate such a grid is given.

\section{A. Stability Criteria}

In order to discuss the robustness against the variation of sampling intervals, we consider the following manipulation of $\Phi$ : Fix $h_{0} \in\left(h_{\ell}, h_{u}\right)$ and then one can define $\theta_{k}$ so that

$$
\tau_{k+1}-\tau_{k}=h_{0}+\theta_{k} .
$$

One has the following property, which is simple but plays a key role in this technical note:

Proposition 1: The function $\Phi(\cdot)$ defined in (6) satisfies

$$
\Phi\left(\tau_{k+1}-\tau_{k}\right)=\Phi\left(h_{0}\right)+\Delta\left(\theta_{k}\right) \Psi\left(h_{0}\right)
$$

where

$$
\Psi(h):=A \Phi(h)+B F, \quad \Delta(\theta):=\int_{0}^{\theta} \mathrm{e}^{A \eta} \mathrm{d} \eta .
$$

Proof: By definition

$$
\Phi\left(\tau_{k+1}-\tau_{k}\right)=\mathrm{e}^{A\left(h_{0}+\theta_{k}\right)}+\int_{0}^{h_{0}+\theta_{k}} \mathrm{e}^{A\left(h_{0}+\theta_{k}-\eta\right)} B \mathrm{~d} \eta F .
$$

The first term can be transformed to

$$
\mathrm{e}^{A\left(h_{0}+\theta_{k}\right)}=\mathrm{e}^{A \theta_{k}} \mathrm{e}^{A h_{0}}=\left(I+\Delta\left(\theta_{k}\right) A\right) \mathrm{e}^{A h_{0}} .
$$

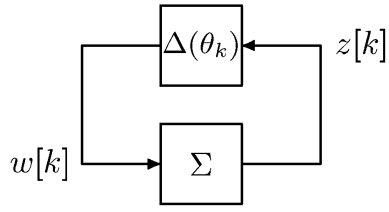

Fig. 2. Alternative representation of $T_{d}$.

While for the second term we have the following:

$$
\begin{aligned}
& \int_{0}^{h_{0}+\theta_{k}} \mathrm{e}^{A\left(h_{0}+\theta_{k}-\eta\right)} B \mathrm{~d} \eta \\
& =\int_{0}^{h_{0}} \mathrm{e}^{A\left(h_{0}+\theta_{k}-\eta\right)} B \mathrm{~d} \eta+\int_{h_{0}}^{h_{0}+\theta_{k}} \mathrm{e}^{A\left(h_{0}+\theta_{k}-\eta\right)} B \mathrm{~d} \eta \\
& =\mathrm{e}^{A \theta_{k}} \int_{0}^{h_{0}} \mathrm{e}^{A\left(h_{0}-\eta\right)} B \mathrm{~d} \eta+\Delta\left(\theta_{k}\right) B \\
& =\left(I+\Delta\left(\theta_{k}\right) A\right) \int_{0}^{h_{0}} \mathrm{e}^{A\left(h_{0}-\eta\right)} B \mathrm{~d} \eta+\Delta\left(\theta_{k}\right) B .
\end{aligned}
$$

Then it is straightforward to derive (8) by substituting the above results.

Now one can regard $T_{d}$ as a feedback connection of an LTI discretetime system $\Sigma$ :

$$
\Sigma[z]:=\Psi\left(h_{0}\right)\left(z I-\Phi\left(h_{0}\right)\right)^{-1}
$$

and a time-varying matrix $\Delta\left(\theta_{k}\right)$. See Fig. 2, where $z[k]=\Psi \xi[k]$ and $w[k]=\Delta\left(\theta_{k}\right) z[k]$. Thus we obtain the following lemma as a simple application of the small-gain theorem: 1

Lemma 2: Let an interval $\mathcal{H} \subseteq(0, \infty)$ be given. There exists a matrix $0<P=P^{*} \in \mathbb{R}^{n \times n}$ satisfying (7) for all $h \in \mathcal{H}$ if $\rho\left(\Phi\left(h_{0}\right)\right)<1$ and

$$
\gamma\|\Delta(\theta)\| \leq 1
$$

for all $\theta \in \mathcal{H}-h_{0}$, where $\gamma$ is an upper bound of $\|\Sigma\|_{\infty}$

$$
\gamma>\|\Sigma\|_{\infty}
$$

Since minimization of $\gamma$ in (11) is routine, one can verify the stability from (10) by bounding $\|\Delta(\theta)\|$. For the purpose we invoke the following property [22]:

Lemma 3: For given $A \in \mathbb{R}^{n \times n}$ and $t \geq 0$ one has

$$
\left\|\mathrm{e}^{A t}\right\| \leq \mathrm{e}^{\mu(A) t}
$$

where $\mu(A)$ denotes the logarithmic norm of $A$ associated with 2-norm

$$
\mu(A)=\lambda_{\max }\left(\frac{A+A^{*}}{2}\right) .
$$

Remark 3: One can continue the following discussion by replacing the bound in (12) by other bounds, that can be found in, e.g., [22], [23].

Remark 4: Instead of the small-gain approach in this technical note, it is suggested in [24], [25] to use the polytopic modeling of $\Delta(\theta)$.

In order to state the main results of this technical note we need the following notation of interval defined with given $h>0$ and $\gamma>0$ :

$$
\mathcal{H}(h, \gamma):=\left[h_{L}, h_{U}\right] \cap(0, \infty)
$$

${ }^{1}$ Readers are referred to, e.g., [21] on the relationship between the quadratic stability and the small-gain condition. 
where $h_{L}$ and $h_{U}$ are given as follows:

L1) If $\mu(-A)=0, h_{L}=h-\gamma^{-1}$;

L2) else if $\mu(-A) \leq-\gamma, h_{L}=-\infty$;

L3) else

$$
h_{L}=h-\frac{1}{\mu(-A)} \log \left(1+\gamma^{-1} \mu(-A)\right) .
$$

U1) If $\mu(A)=0, h_{U}=h+\gamma^{-1}$;

U2) else if $\mu(A) \leq-\gamma, h_{U}=\infty$;

U3) else

$$
h_{U}=h+\frac{1}{\mu(A)} \log \left(1+\gamma^{-1} \mu(A)\right) .
$$

Note that $\mathcal{H}(h, \gamma)$ is non-empty and strictly includes $h$. Now we are ready to state the basic robustness results in this technical note:

Theorem 1: Let $h_{0}>0$ be given so that $\rho\left(\Phi\left(h_{0}\right)\right)<1$. For $\gamma>0$ satisfying (11), there exists a matrix $0<P=P^{*} \in \mathbb{R}^{n \times n}$ satisfying (7) for all $h \in \mathcal{H}\left(h_{0}, \gamma\right)$.

Proof: See Appendix.

\section{B. Algorithm for Stability Analysis}

Theorem 1 provides a robustness condition for $T$ based on the property of the nominal system determined by the fixed sampling period $h_{0}$. A direct use of Theorem 1, however, can be conservative in the sense that there might not exist an $h_{0}>0$ such that $\left[h_{\ell}, h_{u}\right] \subseteq \mathcal{H}\left(h_{0}, \gamma\right)$ even though there exists a matrix $P$ satisfying (7) for all $h \in\left[h_{\ell}, h_{u}\right]$, mainly because of the small-gain type modeling of $\Delta\left(\theta_{k}\right)$.

In order to reduce the conservatism we introduce the multi-model related to $T$ to obtain the following theorem:

Theorem 2: Let $h_{i}>0(i=1,2, \ldots, N)$ be given. If there exist a matrix $0<X=X^{*} \in \mathbb{R}^{n \times n}$ and $\alpha_{i}>0(i=1,2, \ldots, N)$ satisfying $N$ matrix inequalities

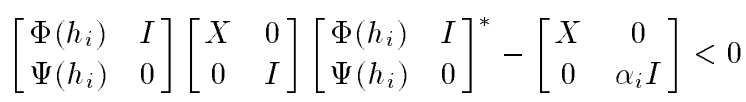

then (7) is satisfied with $P=X^{-1}$ for all

$$
h \in \bigcup_{i=1}^{N} \mathcal{H}\left(h_{i}, \sqrt{\alpha_{i}}\right)
$$

where $\Phi(\cdot), \Psi(\cdot), \mathcal{H}(\cdot, \cdot)$ are defined in (6), (9), and (13), respectively.

Proof: Consider the case $i=1$. The condition (14) with $i=1$ is an equivalent representation of

$$
\left\|\Psi\left(h_{1}\right)\left(z I-\Phi\left(h_{1}\right)\right)^{-1}\right\|_{\infty}<\sqrt{\alpha_{i}} .
$$

Hence, by invoking Theorem 1, there exists a matrix $0<P=P^{*} \in$ $\mathbb{R}^{n \times n}$ satisfying (7) for all $h \in \mathcal{H}\left(h_{1}, \sqrt{\alpha_{1}}\right)$. Moreover we can verify that one of such $P$ is given by $X^{-1}$ from the standard procedure. With similar discussion, we can conclude that there exists a matrix $0<P=$ $P^{*}=X^{-1} \in \mathbb{R}^{n \times n}$ satisfying (7) for all $h \in \mathcal{H}\left(h_{i}, \sqrt{\alpha_{i}}\right), i=$ $2, \ldots, N$. This concludes the proof.

Once we find a matrix $P>0$ satisfying (7) on a grid by any methods, e.g., one proposed in [6], we can verify the robustness by invoking Theorem 2. Moreover, if $T_{d}$ is quadratically stable, there exists a grid verifying it.

Proposition 2: Suppose that there exists a $P=P^{*}>0$ satisfying (7) for all $h \in\left[h_{\ell}, h_{u}\right]$. Then there exists a finite set $\left\{h_{i}\right\}_{i=1}^{N}$ such that there exist $X=X^{*}>0$ and $\alpha_{i}$ 's satisfying (14) and

$$
\left[h_{\ell}, h_{u}\right] \subseteq \bigcup_{i=1}^{N} \mathcal{H}\left(h_{i}, \sqrt{\alpha_{i}}\right)
$$

Proof: The existence of $P$ implies that of an $X=X^{*}>0$ and an $\epsilon>0$ satisfying

$$
\left[\begin{array}{ll}
\Phi(h) & I \\
\Psi(h) & 0
\end{array}\right]\left[\begin{array}{cc}
X & 0 \\
0 & I
\end{array}\right]\left[\begin{array}{ll}
\Phi(h) & I \\
\Psi(h) & 0
\end{array}\right]^{*}-\left[\begin{array}{cc}
X & 0 \\
0 & \left(1 / \epsilon^{2}\right) I
\end{array}\right]<0
$$

for all $h \in\left[h_{\ell}, h_{u}\right]$. Let us consider the case of $\mu(A)>-1 / \epsilon$ and $\mu(-A)>-1 / \epsilon$. Proofs for other cases are easier. We can take $\alpha_{i}=1 / \epsilon^{2}$ for all $i$ 's, and then all the intervals $\mathcal{H}\left(h_{i}, 1 / \epsilon\right)$ have the same width determined by $\mu(A), \mu(-A)$, and $\epsilon$. Hence we can divide $\left[h_{\ell}, h_{u}\right]$ into a finite number of subintervals having width less than $\mathcal{H}\left(h_{i}, 1 / \epsilon\right)$, and thus $(15)$ can be achieved by putting an $h_{i}$ in each subdivision so that $\mathcal{H}\left(h_{i}, 1 / \epsilon\right)$ includes the subdivision.

In the sequel we propose the following concrete algorithm for stability analysis which generates a grid effectively based on Theorem 2:

\section{Algorithm 1}

Given $0<h_{\ell}<h_{u}<\infty$, and a large positive integer $N_{0}$.

0. Initialization: $\mathcal{G} \leftarrow\left\{\left(h_{\ell}+h_{u}\right) / 2\right\}$

1. If there exists an $h \in \mathcal{G}$ satisfying $\rho(\Phi(h)) \geq 1, T$ is unstable. Stop.

2. If $\#(\mathcal{G}) \geq N_{0}$, stop without deciding the stability of $T$. Here $\#(\mathcal{G})$ denotes the number of elements in $\mathcal{G}$.

3. Minimize

$$
{ }_{i=1}^{\#(\mathcal{G})} \frac{\beta_{i}}{\left\|\Sigma_{i}\right\|_{\infty}^{2}}
$$

subject to

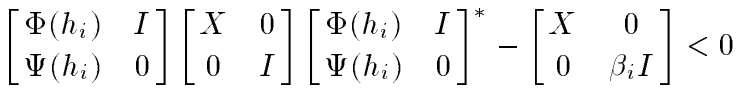

for all $h_{i}$ 's and $X>0$, where

$$
\Sigma_{i}[z]:=\Psi\left(h_{i}\right)\left(z I-\Phi\left(h_{i}\right)\right)^{-1}
$$

and $h_{i}$ is the $i$-th smallest element in $\mathcal{G}$.

4. If

$$
\left[h_{\ell}, h_{u}\right] \subseteq \bigcup_{i=1}^{\#(\mathcal{G})} \mathcal{H}\left(h_{i}, \sqrt{\alpha_{i}}\right)
$$

$T$ is exponentially stable. Stop. Here

$$
\alpha_{i}:=\lambda_{\max }\left(R_{i}-S_{i}^{*}\left(Q_{i}-X_{i}\right)^{-1} S_{i}\right)+\varepsilon
$$

where $\varepsilon$ is a small positive number and

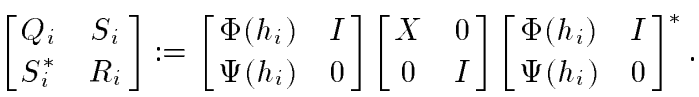

5. Update $\mathcal{G}$ by

$$
\mathcal{G} \leftarrow \mathcal{G} \cup\left\{\left(L_{j}+U_{j}\right) / 2\right\}
$$

for all $j$ where $L_{j}$ and $U_{j}$ are determined so that

$$
\begin{gathered}
\bigoplus_{j=1}^{M}\left(L_{j}, U_{j}\right)=\left(h_{\ell}, h_{u}\right) \backslash\left(\left(h_{\ell}, h_{u}\right) \cap \bigcup_{i=1}^{\#(\mathcal{G})} \mathcal{H}\left(h_{i}, \sqrt{\alpha_{i}}\right)\right), \\
L_{1}<U_{1}<L_{2}<U_{2}<\cdots<L_{M}<U_{M}
\end{gathered}
$$

are satisfied. Go to Step 1 . Here the symbol $\oplus$ denotes the direct sum. 
We have some remarks for Algorithm 1: Step 2 is introduced to avoid numerical issues which could happen when \#(G) is too large, and Algorithm 1 stops after $N_{0}-1$ iterations at most. The performance of the algorithm can be tuned by modifying the objective function in Step 3 so that the stopping criterion in Step 4, which is a nonlinear condition, is satisfied effectively. Note that $\alpha_{i}$ satisfies (14) with $X$ determined in Step 3 and $\alpha_{i} \leq \beta_{i}$ with sufficiently small $\varepsilon$. The integer $M$ in Step 5 is $\#(\mathcal{G})+1$ at most.

\section{NUMERICAL EXAMPLES}

In this section we demonstrate the validity of the proposed method for stability analysis. More numerical examples can be found in the conference version of this technical note [26].

Let us consider the following second-order problem parameters [6]:

$$
A=\left[\begin{array}{cc}
0 & 1 \\
0 & -0.1
\end{array}\right], \quad B=\left[\begin{array}{c}
0 \\
0.1
\end{array}\right], \quad F=-\left[\begin{array}{ll}
3.75 & 11.5
\end{array}\right]
$$

We search for an interval $\tilde{\mathcal{H}}$ satisfying that $T$ is exponentially stable if

$$
\tau_{k+1}-\tau_{k} \in \tilde{\mathcal{H}}
$$

Applying the methods in the literature we obtain several $\tilde{\mathcal{H}}$ 's: From the results in [4] we have $\left(0,2.7 \times 10^{-4}\right]$ (reported in [5]). It is improved to $\left(0,4.5 \times 10^{-4}\right]$ in [5], and $(0,0.0593]$ in [6]. A significant improvement is done in [7], [9] to have $(0,0.869]$. One can find further improvements: $(0,1.113]$ in [11], [12], and $(0,1.365]$ in [13].

It is natural to take $h_{\ell}>0$ for practical situations of networked/embedded control systems. Here let us take $h_{\ell}=0.01$. Then the proposed algorithm proved the exponential stability of $T$ for $\tilde{\mathcal{H}}=[0.01,1.72]$ with

$$
P=\left[\begin{array}{cc}
4.03 & 5.09 \\
5.09 & 13.49
\end{array}\right] \times 10^{-3}
$$

We remark that $T$ is unstable when the sampling period is fixed to 1.73.

We have implemented Algorithm 1 on MATLAB 7.4, YALMIP (R20070523) [27], and SDPT3 (4.0 3 ) [28]. The search took 6.33 [s] on a laptop with Intel Core 2 Duo $(2.33 \mathrm{GHz})$ running MacOSX, and the maximal \# $(\mathcal{G})$ in the search was 25 .

\section{EXTENSIONS FOR CONSERVATISM REDUCTION}

The proposed algorithm chops the given interval $\left[h_{\ell}, h_{u}\right]$ into pieces to verify the stability by using Theorem 1 . Although it enables to test the stability for large range of the sampling interval in spite of the conservatism in Theorem 1, it is obvious that the performance of the algorithm is improved if one can reduce the conservatism in Theorem 1. There are several directions for the purpose. In this section we suggest and discuss some of them with numerical evaluation.

A straightforward way is to replace the bound of the maximal singular value of matrix exponential in (12) by other bounds found in, e.g., [22], [23]. Since the performance of the bound depends on the matrix taken the exponential [22], [23], which is the ' $A$ '-matrix of the plant in our problem, it might be practical to use bounds as many as possible and take the least conservative one if computational time is allowed.

Another way to reduce the conservatism is to replace the small gain condition (10) by a general quadratic condition in, e.g., [29], [30]. For the generalization it is required to find a matrix $\Pi=\Pi^{*} \in \mathbb{R}^{(n+m) \times(n+m)}$ satisfying

$$
I_{\Delta}^{*} \Pi^{*} \begin{aligned}
& I \\
& \Delta
\end{aligned} \geq 0
$$

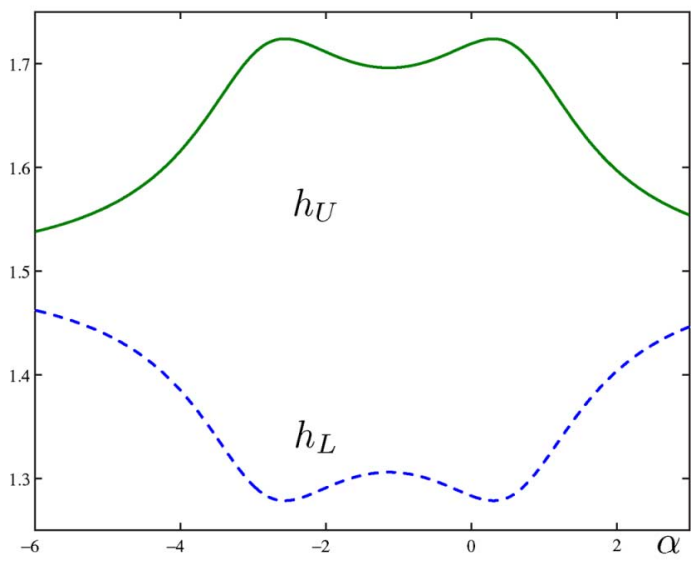

Fig. 3. $h_{U}$ and $h_{L}$ varying $\alpha$.

for all $\Delta \in\left\{\Delta(\theta), \theta \in\left[h_{\ell}-h_{0}, h_{u}-h_{0}\right]\right\}$. One such $\Pi$ is given by

$$
\Pi=\left[\begin{array}{cc}
\mathrm{e}^{-A^{*} \alpha} \mathrm{e}^{-A \alpha} & 0 \\
0 & -\gamma^{2} \mathrm{e}^{-A^{*} \alpha} \mathrm{e}^{-A \alpha}
\end{array}\right]
$$

for all $\alpha \in \mathbb{R}$ and $\gamma \leq 1 /\|\Delta\|$, noting that $\Delta(\theta)$ and $\mathrm{e}^{A \alpha}$ commute. Note that this $\Pi$ is related to the scaled small gain condition and one can reduce the conservatism in Theorem 1 by replacing $\|\Sigma\|_{\infty}$ by $\left\|\mathrm{e}^{-A \alpha} \Sigma \mathrm{e}^{A \alpha}\right\|_{\infty}$. For the problem data in Section IV with $h_{0}=$ 0.1 , Theorem 1 and the above condition verify the stability for $h_{0}+$ $[-6.85,6.87] \times 10^{-2}$ and $h_{0}+[-9.52,9.57] \times 10^{-2}$ respectively, where we swept $\alpha$ from -6 to 3 with 0.01 step. This is more than $35 \%$ improvement. One drawback of this method is the fact that the corresponding optimization problem is not convex: For the problem data in Section IV with $h_{0}=1.5$, we plot

$$
\left[h_{L}, h_{U}\right]=\mathcal{H}\left(h_{0}, \gamma\right)
$$

for $\gamma>\left\|\mathrm{e}^{-A \alpha} \Sigma \mathrm{e}^{A \alpha}\right\|_{\infty}$ in Fig. 3, varying $\alpha$. We see that both $h_{L}$ and $h_{U}$ are multimodal in $\alpha$.

\section{CONCLUSION}

We have considered stability of sampled-data feedback control systems where the state is sampled aperiodically, motivated by widespread use of networked and embedded control systems.

We have proposed a stability analysis algorithm by showing robustness of sampled-data systems against perturbation caused by variation of sampling intervals based on the small-gain framework. We have also discussed some directions for reducing the conservatism.

In this technical note we have considered an analysis problem for a simple sampled state feedback scenario, however, application to more practical analysis and synthesis problems are not hard and have been partially done in [31], [32].

\section{APPENDIX}

Proof of Theorem 1: We here prove that (10) holds for all $h \in$ $\left[h_{0}, h_{U}\right]$. The proof for the interval $\left[h_{L}, h_{0}\right]$ is similar so it is omitted. Note that $H\left(h_{0}, \gamma\right) \subseteq\left[h_{L}, h_{U}\right]$.

Invoking Lemma 3 we have

$$
\|\Delta(\theta)\| \leq \int_{0}^{\theta}\left\|\mathrm{e}^{A t}\right\| \mathrm{d} t \leq \int_{0}^{\theta} \mathrm{e}^{\mu(A) t} \mathrm{~d} t
$$

when $\theta \geq 0$. If $\mu(A)=0$

$$
\|\Delta(\theta)\| \leq \theta .
$$


Hence (10) holds as long as $\gamma \theta \leq 1$. This completes the proof for the case U1.

Let us next consider the case of $\mu(A) \neq 0$. In this case

$$
\|\Delta(\theta)\| \leq \frac{\mathrm{e}^{\mu(A) \theta}-1}{\mu(A)} .
$$

Suppose that $\mu(A)<0$. Noting that the right hand side goes to $-1 / \mu(A)$ when $\theta$ tends to $\infty$. Hence (10) holds for all $\theta>0$ if

$$
-\frac{\gamma}{\mu(A)} \leq 1
$$

This completes the proof for the case $\mathrm{U} 2$.

Finally let us consider the case of $\mu(A) \neq 0$ and

$$
-\frac{\gamma}{\mu(A)}>1
$$

The small gain condition (10) holds for all $\theta>0$ if

$$
\gamma \frac{\mathrm{e}^{\mu(A) \theta}-1}{\mu(A)} \leq 1 .
$$

Noting that $1+\gamma^{-1} \mu(A)>0$ in this case, this condition turns to

Case A) If $\mu(A)>0$

$$
\mu(A) \theta \leq \log \left(1+\gamma^{-1} \mu(A)\right) .
$$

Case B) If $\mu(A)<0$

$$
\mu(A) \theta \geq \log \left(1+\gamma^{-1} \mu(A)\right) .
$$

Hence, we have

$$
\theta \geq \frac{1}{\mu(A)} \log \left(1+\gamma^{-1} \mu(A)\right) .
$$

for both cases. This completes the proof for the case U3.

\section{ACKNOWLEDGMENT}

The author would like to express his appreciations to Prof. L. Mirkin who introduced the author to this subject with fruitful discussions, and the anonymous referees for pointing out recent references.

After the conference presentation [26], we have received closely related results [33], [34].

\section{REFERENCES}

[1] T. Chen and B. Francis, Optimal Sampled-Data Control Systems. New York: Springer, 1995.

[2] , D. Hristu-Varsakelis and W. Levine, Eds., Handbook of Networked and Embedded Control Systems. Boston, MA: Birkhäuser, 2005.

[3] J. Hespanha, P. Naghshtabrizi, and Y. Xu, "A survey of recent results in networked control systems," Proc. IEEE, vol. 95, no. 1, pp. 138-162, Jan. 2007.

[4] G. Walsh, H. Ye, and L. Bushnell, "Stability analysis of networked control systems," in Proc. Amer. Control Conf., 1999, pp. 2876-2880.

[5] W.Zhang, M. Branicky, and S. Phillips, "Stability of networked control systems," IEEE Control Syst. Mag., vol. 21, pp. 84-99, 2001.

[6] W. Zhang and M. Branicky, "Stability of networked control systems with time-varying transmission period," in Proc. Conf. Commun., Contr. Comput., 2001, [CD ROM].

[7] E. Fridman, A. Seuret, and J. Richard, "Robust sampled-data stabilization of linear systems: An input delay approach," Automatica, vol. 40, pp. 1441-1446, 2004.

[8] E. Fridman, U. Shaked, and V. Suplin, "Input/output delay approach to robust sampled-data $H_{\infty}$ control," Syst. Control Lett., vol. 54, pp. 271-282, 2005.

[9] P. Naghshtabrizi and J. Hespanha, "Designing an observer-based controller for a network control system," in Proc. 44th IEEE Conf. Decision Control, Eur. Control Conf., 2005, pp. 848-853.
[10] V. Suplin, E. Fridman, and U. Shaked, "Sampled-data $H_{\infty}$ control and filtering: Nonuniform uncertain sampling," Automatica, vol. 43 , pp. 1072-1083, 2007.

[11] P. Naghshtabrizi, J. Hespanha, and R. Teel, "On the robust stability and stabilization of sampled-data systems: A hybrid system aproach," in Proc. 45th IEEE Conf. Decision Control, 2006, pp. 4873-4878.

[12] P. Naghshtabrizi, J. Hespanha, and A. Teel, "Stability of delay impulsive systems with application to networked control systems," in Proc. Amer. Control Conf., 2007, pp. 4899-4904.

[13] L. Mirkin, "Some remarks on the use of time-varying delay to model sample-and-hold circuits," IEEE Trans. Autom. Control, vol. 52, no. 6 , pp. 1109-1112, Jun. 2007.

[14] H. Fujioka, "Stability analysis of systems with aperiodic sample-andhold devices," Automatica, vol. 45, pp. 771-775, 2009.

[15] A. Sala, "Computer control under time-varying sampling period: An LMI gridding approach," Automatica, vol. 41, pp. 2077-2082, 2005.

[16] G. Walsh, O. Beldiman, and L. Bushnell, "Asymptotic behavior of nonlinear networked control systems," IEEE Trans. Autom. Control, vol. 46, no. 7, pp. 1093-1097, Jul. 2001.

[17] D. Carnevale, A. Teel, and D. Nešić, "A Lyapunov proof of an improved maximum allowable transfer interval for networked control systems," IEEE Trans. Autom. Control, vol. 52, no. 5, pp. 892-897, May 2007.

[18] M. Cloosterman, N. van de Wouw, W. Heemels, and H. Nijmeijer, "Stabilization of networked control systems with large delays and packet dropouts," in Proc. Amer. Control Conf., 2008, pp. 4991-4996.

[19] Y. Pan, H. Marquez, and T. Chen, "Remote stabilization of networked control systems with unknown time varying delays by LMI techniques," in Proc. 44th IEEE Conf. Decision Control, Eur. Control Conf., 2005, pp. 1589-1594.

[20] L. Hetel, J. Daafouz, and C. Iung, "Stabilization of arbitrary switched linear systems with unknown time-varying delays," IEEE Trans. Autom. Control, vol. 51, no. 10, pp. 1668-1674, Oct. 2006.

[21] P. Khargonekar, I. Petersen, and K. Zhou, "Robust stabilization of uncertain linear systems: Quadratic stabilizability and $H^{\infty}$ control theory," IEEE Trans. Autom. Control, vol. 35, no. 3, pp. 356-361, Mar. 1990.

[22] C. V. Loan, "The sensitivity of the matrix exponential," SIAM J. Numer. Anal., vol. 14, pp. 971-981, 1977.

[23] B. Kågström, "Bounds and perturbation bounds for the matrix exponential," BIT, vol. 17, pp. 89-97, 1977.

[24] A. Balluchi, P. Murrieri, and A. Sangiovanni-Vincentelli, "Controller synthesis on non-uniform and uncertain discrete-time domains," in $\mathrm{Hy}$ brid Systems: Computation and Control. New York: Springer, 2005.

[25] L. Hetel, J. Daafouz, and C. Iung, "Analysis and control of LTI and switched systems in digital loops via an event-based modelling," Int. J. Control, vol. 81, pp. 1125-1138, 2008.

[26] H. Fujioka, "Stability analysis for a class of networked/embedded control systems: A discrete-time approach," in Proc. Amer. Control Conf., 2008, pp. 4997-5002.

[27] J. Löfberg, "YALMIP: A toolbox for modeling and optimization in MATLAB," in Proc. IEEE CACSD Conf., 2004, pp. 284-289.

[28] R. Tutuncu, K. Toh, and M. Todd, "Solving semidefinite-quadraticlinear programs using SDPT3," Math. Programming Ser. B, vol. 95, pp. 189-217, 2003

[29] A. Megretski and A. Rantzer, "System analysis via integral quadratic constraints," IEEE Trans. Autom. Control, vol. 42, no. 6, pp. 819-830, Jun. 1997.

[30] T. Iwasaki and S. Hara, "Well-posedness of feedback systems: Insights into exact robustness analysis and approximate computations," IEEE Trans. Autom. Control, vol. 48, no. 5, pp. 619-630, May 1998.

[31] H. Fujioka, "Stability analysis for a class of networked/embedded control systems: Output feedback case," in Proc. 17th IFAC World Congress, 2008, pp. 4210-4215.

[32] H. Fujioka and T. Nakai, "Stabilizing systems with aperiodic sample-and-hold devices," in Proc. UKACC Int. Conf. Control, 2008, [CD ROM].

[33] Y. Suh, "Stability and stabilization of nonuniform sampling systems," Automatica, vol. 44, pp. 3222-3226, 2008.

[34] J. Skaf and S. Boyd, "Analysis and synthesis of state-feedback controllers with timing jitter," IEEE Trans. Autom. Control, vol. 54, no. 3, pp. 652-657, Mar. 2009. 